\title{
Speckle reduction using the discrete Fourier filtering technique
}

\author{
Jonathan Maycock, Bryan M. Hennelly, John B. McDonald, Dept. CS, NUI Maynooth \\ Yann Frauel, Dept. de Ciencias de la Computación, Univ. Nacional Autónoma de México \\ Albertina Castro, Instituto Nacional de Astrofísica, Óptica y Electrónica, México \\ Bahram Javidi, Dept. of Elec. and Comp. Eng., Univ. of Connecticut \\ Thomas J. Naughton, Dept. CS, NUI Maynooth and Univ. Of Oulo, Finland \\ tomn@cs.nuim.ie
}

\begin{abstract}
We present a digital signal processing technique that reduces the speckle content in reconstructed digital holograms. The method is based on sequential sampling of the discrete Fourier transform of the reconstructed image field. The resulting images show a reduction in speckle.
\end{abstract}

\section{Introduction}

Holography is an established technique for recording and reconstructing real-world 3D objects. Digital holography has become feasible owing to recent advances in megapixel CCD sensors with high spatial resolution and high dynamic range. Speckle occurs when coherent light is diffused by an optically rough surface. It degrades the quality of the rendered images and makes the accurate viewing of small details difficult. The speckle problem is always present in holography since it uses coherent light.

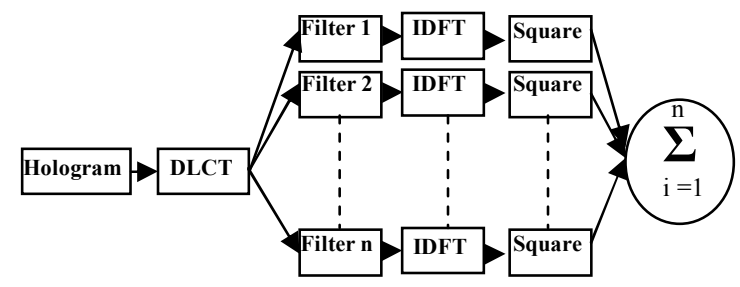

Figure 1. A schematic of the DFF technique. DLCT, discrete linear canonical transform; IDFT, inverse discrete Fourier transform.

\section{The technique}

We have developed the discrete Fourier filter (DFF) technique (see Fig. 1) and applied it to reconstructions of digital holograms (DHs). The first step is to numerically compute the propagation from the discrete hologram plane to the discrete Fourier plane. The Fourier plane data is filtered and then inverse discrete Fourier transformed to the image plane where its intensity is stored. This is repeated $n$ times and the resulting $n$ intensities are summed. In [1] it was shown that as long as two positions of the aperture do not overlap, the power spectrum of the speckle is reduced to half its value.

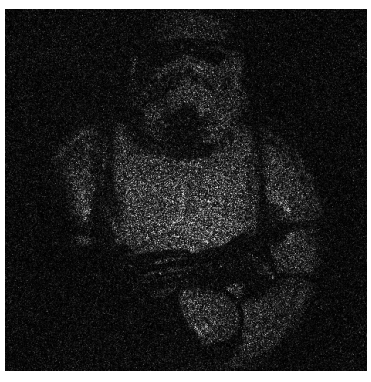

(a)

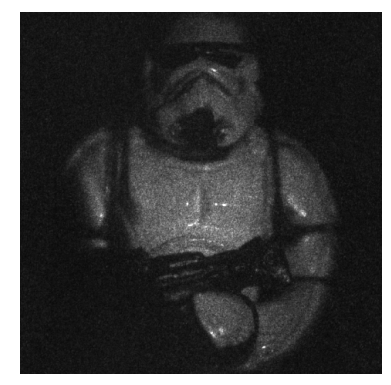

(b)
Figure 2. (a) Shows the original reconstruction, and, (b) shows the result of applying the DFF technique.

\section{Results}

A well known metric called speckle index was used to measure the reduction in speckle. The lower the speckle index the lower the speckle content. In Fig. 2 (a), the original reconstruction is shown. It is $2048 \mathrm{x}$ 2048 pixels in size, and has a speckle index of approximately 1 . The application of the DFF to a reconstruction of this DH is shown in Fig. 2(b). The aperture used was $256 \times 256$ pixels in size. This results in a reduced speckle index of 0.2 . See [1] for a full analysis of the technique and comparisons to the mean and median filters in terms of speckle index and resolution.

\section{References}

[1] J. Maycock, B. M. Hennelly, J. B. Mc Donald, T. J. Naughton, Y. Frauel, A. Castro, and B. Javidi. Reduction of speckle in digital holography by discrete Fourier filtering. J. Opt. Soc. Am. A, 24:1617-1622, 2007. 